\title{
Influence of microstructure on symmetry determination of piezoceramics
}

\author{
M. Hinterstein, H. E. Mgbemere, M. Hoelzel, W. Rheinheimer, E. \\ Adabifiroozjaei, P. Koshy, C. C. Sorrell and M. Hoffman
}

J. Appl. Cryst. (2018). 51, 670-678

\section{IUCr Journals CRYSTALLOGRAPHY JOURNALS ONLINE}

Copyright (C) International Union of Crystallography

Author(s) of this article may load this reprint on their own web site or institutional repository provided that this cover page is retained. Republication of this article or its storage in electronic databases other than as specified above is not permitted without prior permission in writing from the IUCr.

For further information see http://journals.iucr.org/services/authorrights.html 
JOURNAL OF

APPLIED

CRYSTALLOGRAPHY

ISSN 1600-5767

Received 1 August 2017

Accepted 6 March 2018

Edited by A. J. Allen, National Institute of Standards and Technology, Gaithersburg, USA

Keywords: piezoceramics; X-ray diffraction; neutron diffraction; potassium sodium niobate; microstructure.

CCDC references: $1828074 ; 1828075$; 1828076 ; 1828077; 1828078; 1828079; $1828080 ; 1828081 ; 1828082 ; 1828083$

Supporting information: this article has supporting information at journals.iucr.org/j

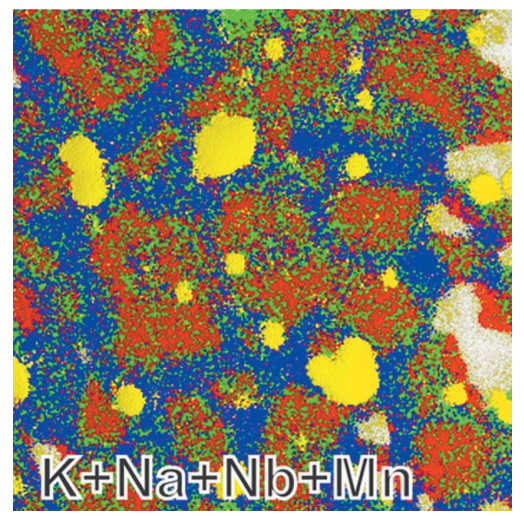

(C) 2018 International Union of Crystallography

\section{Influence of microstructure on symmetry determination of piezoceramics}

\author{
M. Hinterstein, ${ }^{\mathrm{a}, \mathrm{b} *}$ H. E. Mgbemere, ${ }^{\mathrm{c}}$ M. Hoelzel, ${ }^{\mathrm{d}}$ W. Rheinheimer, ${ }^{\mathrm{a}}$ \\ E. Adabifiroozjaei, ${ }^{\text {b }}$ P. Koshy, ${ }^{\text {b }}$ C. C. Sorrell ${ }^{b}$ and M. Hoffman ${ }^{b}$
}

\begin{abstract}
${ }^{\mathbf{a}}$ Institute for Applied Materials, Karlsruhe Institute of Technology, 76131 Karlsruhe, Germany, ${ }^{\mathbf{b}_{\text {School }}}$ of Materials Science and Engineering, UNSW Sydney, Sydney, Australia, '⿳亠口冋epartment of Metallurgical and Materials Engineering, University of Lagos, Akoka, Lagos, Nigeria, and 'Heinz Maier-Leibnitz Zentrum, Technische Universität München, Garching, Germany. *Correspondence e-mail: m.hinterstein@kit.edu
\end{abstract}

The origin of the complex reflection splitting in potassium sodium niobate doped with lithium and manganese was investigated using temperaturedependent high-resolution X-ray and neutron diffraction as well as electron probe microanalysis and scanning electron microscopy. Two structural models were developed from the diffraction data. A single-phase monoclinic Pm model is known from the literature and is able to reproduce the diffraction patterns perfectly. However, a model with phase coexistence of two classical orthorhombic Amm2 phases can also reproduce the diffraction data with equal accuracy. Scanning electron microscopy in combination with electron probe microanalysis revealed segregation of the $A$-site substituents potassium and sodium. This favours the model with phase coexistence and confirms the need for comprehensive analyses with complementary methods to cover a broad range of length scales as well as to assess both average and local structure.

\section{Introduction}

The influence of the microstructure on diffraction patterns is a widely discussed issue in materials science. For piezoceramics in particular, not only the grain morphology but also the microstructure of the grains themselves play crucial roles. The more common material systems, such as lead zirconate titanate (PZT) (Jaffe et al., 1971) and barium titanate (BT) (Kay \& Vousden, 1949), exhibit complex domain structures, especially in the vicinity of the phase boundaries (Schönau et al., 2007; Theissmann et al., 2007; Forsbergh, 1949). These complex structures cause anisotropic reflection broadening since the domain walls interrupt the ideal structure and prevent the development of a homogeneous crystal structure throughout the grains (Boysen, 2007, 2005).

With the improvement of diffraction techniques, the phase diagrams of many piezoelectric systems have been revised (Baker et al., 2009; Cox et al., 2001; Keeble et al., 2013; LaOrauttapong et al., 2002; Zhang et al., 2014; Tellier et al., 2009; Ranjan, Mishra \& Pandey, 2002; Zhang et al., 2011a,b). While most systems can be characterized in terms of rhombohedral, orthorhombic or tetragonal phases, high-resolution measurements have revealed the existence of monoclinic phases (Noheda et al., 1999; Gorfman \& Thomas, 2010; Mgbemere et al., 2012). For PZT, a monoclinic $C m$ phase has been proposed at the phase boundary between the tetragonal $P 4 \mathrm{~mm}$ and the rhombohedral $R 3 m$ phases above room temperature (Noheda et al., 1999). Below room temperature the $R 3 \mathrm{~m}$ phase transforms to $R 3 c$ with oxygen octahedral tilting. This low- 
temperature modification subsequently was found to exhibit monoclinic $C c$ symmetry instead of rhombohedral $R 3 c$ symmetry (Mishra et al., 2001).

In the lead-free bismuth sodium titanate (BNT) system, the rhombohedral $R 3 c$ phase was reported to be of monoclinic $C c$ symmetry (Aksel et al., 2011) and this was confirmed with single-crystal diffraction (Gorfman \& Thomas, 2010). A similar discussion remains pending for the potassium sodium niobate (KNN) system, which is known to exhibit a challenging processing behaviour (Malič et al., 2015). For the composition $\mathrm{K}_{0.5} \mathrm{Na}_{0.5} \mathrm{NbO}_{3}$, there remains uncertainty whether the structure is orthorhombic Amm2 (Mgbemere et al., 2011) or monoclinic Pm (Tellier et al., 2009). However, this represents a special case since $A m m 2$ does not describe a primitive cell; it contains two formula units and two of the axes are primitive $[110]_{\mathrm{C}}$ directions. Different lattice parameters for these two axes result in a rhombic distortion of the (001) plane, giving the primitive cell monoclinic symmetry with equal $a$ and $b$ axes (Mgbemere et al., 2012). In contrast, the Pm structure is set up as a primitive cell, resulting in the possibility of having three different lengths for the primitive axes (Mgbemere et al., 2012).

The rationale for the preceding cases often is based on the agreement factors for Rietveld refinements (Pandey \& Agini, 2003; Aksel et al., 2011; Ranjan, Mishra, Pandey \& Kennedy, 2002; Ranjan, Mishra \& Pandey, 2002; Zhang et al., 2011a,b). Since the complex real structure generates anisotropic reflection broadening, the increased number of reflections representing the monoclinic phases assists in modelling the measured profiles. Only a few studies have provided evidence of lower symmetry by clear reflection splitting (Gorfman \& Thomas, 2010; Mgbemere et al., 2012; Noheda et al., 1999).

For undoped PZT, a clear additional reflection has suggested a monoclinic modification (Noheda et al., 1999). However, other studies have attempted to explain this observation by either coherent scattering of the nanodomains or phase coexistence (Wang, 2007). It is only with BNT that a single-crystal diffraction experiment has demonstrated unambiguously the existence of monoclinic reflection splitting (Gorfman \& Thomas, 2010). Furthermore, only with powder diffraction data has pronounced reflection broadening been observed (Aksel et al., 2011).

All high-performance piezoceramics are located near phase boundaries in systems exhibiting solid solubility. The best known system is PZT with $B$-site substitution of zirconium by titanium (Jaffe et al., 1971). Both BNT and KNN are $A$-site substituted while, for BT, a typical combination includes $A$ and $B$-site substitutions with $\mathrm{Ca}$ and $\mathrm{Zr}$, respectively, to form the complex system $(1-x) \mathrm{Ba}\left(\mathrm{Zr}_{y} \mathrm{Ti}_{1-y}\right) \mathrm{O}_{3-x}\left(\mathrm{Ba}_{z} \mathrm{Ca}_{1-z}\right) \mathrm{TiO}_{3}$ (Keeble et al., 2013). Grinberg et al. (2002) showed by density functional theory calculations that the distribution of the substituted species influences the local structure of the material.

The present study demonstrates the challenges of structure determination for KNN-based functional piezoceramics. The complex microstructure results in diffraction patterns that can be interpreted in terms of low symmetry or phase coexistence.
On the basis of agreement factors alone, neither of the models can be considered as definitive. However, a supported conclusion can be drawn using a combination of microstructural and elemental analytical methods.

\section{Experimental}

$\mathrm{KNN}-\mathrm{Li}_{2} \mathrm{Mn} \quad\left[\left(\mathrm{K}_{0.48} \mathrm{Na}_{0.48} \mathrm{Li}_{0.04}\right)\left(\mathrm{Nb}_{0.98} \mathrm{Mn}_{0.02}\right) \mathrm{O}_{3}\right]$ was produced from $\mathrm{K}_{2} \mathrm{CO}_{3}, \mathrm{Na}_{2} \mathrm{CO}_{3}, \mathrm{Li}_{2} \mathrm{CO}_{3}$ (all 99 wt\%), $\mathrm{Nb}_{2} \mathrm{O}_{5}$ (99.9\%) (Chempur Feinchemikalien und Forschungs $\mathrm{GmbH}$, Karlsruhe, Germany) and $\mathrm{MnO}_{2}$ (95\%) (Merck Chemicals Darmstadt, Germany). The powders were dried at $493 \mathrm{~K}$ for $4 \mathrm{~h}$ in order to remove moisture. Stoichiometric amounts of the starting oxides were batched, but a $2 \mathrm{~mol} \%$ excess of the carbonates was used in order to compensate for losses during sintering (Ahn \& Schulze, 1987; Bomlai et al., 2007). The powders were mixed and milled using an attrition mill (NETZSCH-Feinmahltechnik GmbH) operating at $500 \mathrm{r} \mathrm{min}^{-1}$ for $2 \mathrm{~h}$ with ethanol as medium and $3 \mathrm{~mm}$ yttria-stabilized zirconia balls as grinding media. The solvent (ethanol) was removed using a rotary evaporator (Rotavapor R-100 BÜCHI Labortechnik AG, Flawil, Switzerland), after which the powders were placed in alumina crucibles and calcined in a tube furnace at $1123 \mathrm{~K}$ for $4 \mathrm{~h}$ at a heating rate of $3 \mathrm{~K} \mathrm{~min}^{-1}$, with subsequent cooling at $10 \mathrm{~K} \mathrm{~min}^{-1}$. Following calcination, the attrition milling process was repeated in order to homogenize the powders and to reduce the average particle size of the powders to $<1 \mu \mathrm{m}$. The powders were then cold isostatically pressed (wet-bag method) at $300 \mathrm{MPa}$ for $2 \mathrm{~min}$. The samples were placed on alumina supports and sintered in air at $1323 \mathrm{~K}$ for $1 \mathrm{~h}$, using a heating rate of $2 \mathrm{~K} \mathrm{~min}^{-1}$ and a cooling rate of $10 \mathrm{~K} \mathrm{~min}^{-1}$.

High-resolution synchrotron X-ray powder diffraction data were obtained using flat-plate transmission geometry at the B2 beamline at Deutsches Elektronen-Synchrotron (DESY) in Hamburg, Germany (Knapp et al., 2004), using the analyser crystal detector at a beam energy of $18 \mathrm{keV}(\lambda=0.6881 \AA)$ with a step width of $0.002^{\circ} 2 \theta$. High-resolution neutron powder diffraction data were obtained from a sintered cylindrical sample of $\sim 400 \mathrm{~mm}^{3}$ using the SPODI beamline at the Maier Leibnitz-Zentrum in Garching, Germany (Hoelzel et al., 2012). This beamline operates at a wavelength $\lambda=$ $1.548 \AA$ using a bank of 80 position-sensitive ${ }^{3} \mathrm{He}$ detectors that cover a $160^{\circ}$ scattering range. High-temperature measurements were performed in situ in a high-temperature furnace in the range 300-500 K. Since the furnace elements consisted of niobium, it was necessary to perform the heating under vacuum $\left(10^{-5} \mathrm{mbar} \equiv 10 \mathrm{mPa}\right)$ in order to prevent oxidation.

Rietveld refinement was performed using the Fullprof software package (Rodríguez-Carvajal, 1993). The structure models consisted of different phases with rhombohedral $(R 3 c)$, monoclinic $(P m)$, orthorhombic (Amm2), tetragonal $(P 4 m m)$ and cubic $(P m \overline{3} m)$ symmetry known from the literature. The lithium ions were refined on the $A$ site together with potassium and sodium, and the manganese ions were refined on the $B$ site together with niobium. In order to obtain 
Table 1

Details of the applied structure models of the refinements against neutron (ND) and X-ray (XRD) diffraction data.

The columns give the total number of parameters (Par.), goodness of fit $(\mathrm{GoF})$, least-squares agreement factor $\left(\chi^{2}\right)$, the number of refined parameters belonging to the atomic (at.), lattice (la.), profile (pr.), background (ba.) and other (ot.) parameter sets, the number of refined parameters belonging to common (co.) and individual (in.) parameter sets, and the number of calculated reflections (Ref.).

\begin{tabular}{llllllllllllllll}
\hline Source & Model & $T(\mathrm{~K})$ & Par. & GoF & $\chi^{2}$ & at. & la. & pr. & ba. & ot. & co. & in. & Ref. \\
\hline ND+XRD & $P m$ & 300 & 69 & 75 & 4.86 & 11 & 4 & 33 & 16 & 5 & 21 & 48 & 287 \\
ND+XRD & Amm 2 & 300 & 66 & 100 & 4.78 & 14 & 6 & 27 & 16 & 5 & 21 & 47 & 346 \\
ND & $P 4 m m$ & 526 & 41 & 411 & 12.1 & 14 & 4 & 10 & 10 & 3 & 13 & 28 & 112 \\
ND & $P m \overline{3} m$ & 776 & 26 & 540 & 15.7 & 6 & 2 & 5 & 10 & 3 & 13 & 13 & 52 \\
\hline
\end{tabular}

consistent refinement results, similar sets of parameters were used for all refinements. The refined parameters can be categorized in five different groups: atomic, lattice, profile, background and others. The number of parameters in categories 'background' and 'others' was always the same for all refinements. These can be considered as a 'common' set of parameters. Category 'others' consists of zero shift and scale factors for the individual phases. The 'background' was refined using linear interpolation between an identical set of points from the regions where no reflections contributed to the intensities.

The numbers of parameters in categories 'atomic', 'lattice' and 'profile' depend on the symmetry and these are considered as 'individual' sets of parameters. Category 'profile' consists of various parameters for reflection broadening due to strain effects, which are common in piezoceramics. The profile function was described using the Thompson-Cox-Hastings pseudo-Voigt model (Thompson et al., 1987). The integral breadth was calculated after subtraction of instrumental broadening and the implementation of a model for anisotropic peak broadening, developed by Stephens (1999). The number of possible parameters in this model depends on symmetry, and the model considers the case of an anisotropic distribution of strain, in which the diffraction peak width increases in proportion to the diffraction order. The instrumental broadening was determined by a Rietveld fit of a high-resolution measurement recorded at ambient temperature of the standard reference material $\mathrm{LaB}_{6}$ (NIST SRM 660a) for X-ray measurements and NAC $\left(\mathrm{Na}_{2} \mathrm{Ca}_{3} \mathrm{Al}_{2} \mathrm{~F}_{14}\right)$ for neutron measurements. Category 'lattice' consists of the lattice parameters and category 'atomic' consists of positions and DebyeWaller factors of the individual atoms in the structure model. Both sets of parameters are dependent on symmetry. The difference between $\mathrm{Na}$ and $\mathrm{K}$ is essentially invisible for both $\mathrm{X}$-rays and neutrons owing to the very similar atom form factors and scattering lengths in X-ray and neutron diffraction. Therefore, the occupation of the $A$ sites was not refined.

The ambient-temperature refinements were performed against both X-ray and neutron data simultaneously in a single fit with a 1:1 weighting of the data sets. In order to reduce the number of refined parameters, atomic parameters, scale factors and lattice parameters were constrained between $\mathrm{X}$-ray and neutron structure models. For any two-phase structure model the atomic, lattice and profile parameters were refined individually for each phase. An overview of the different settings for the applied structure models can be found in Table 1.

The elemental distributions were analysed by electron probe microanalysis (EPMA) using a JEOL JXA-8500F (Tokyo, Japan) instrument equipped with four wavelengthdispersive spectrometers that were individually optimized for the detection of the different elements. Quantitative microstructural analysis of the EPMA scans was performed using ImageJ (Schneider et al., 2012).

\section{Results}

\subsection{Powder diffraction}

The authors recently published a report on doped KNN that showed clear reflection splitting (Mgbemere et al., 2012). The data were modelled unambiguously with a monoclinic $P m$ structure, the evidence for which is shown in Fig. 1(a). There also is direct evidence of reflection splitting from the monoclinic angle, for example for the $\overline{1} 10_{\mathrm{M}}$ reflection [arrow in Fig. 1(a), inset I], and the three different axial lengths of the primitive unit cell, for example for the $200_{\mathrm{C}}$ reflection [Fig. 1(a), inset II]. Although the insets of Fig. 1(a) indicate imperfect agreement between the measured data and the fit, closer examination suggests that the cause is the complicated profile and anisotropic reflection broadening due to microstrain. Analysis of the reflection widths by Rietveld refinement confirms this assessment and reveals the strongest anisotropy for the $200_{C}$ reflection, with microstrains of $0.104 \%$ for the $020_{\mathrm{M}}$ reflection, $0.238 \%$ for the $002_{\mathrm{M}}$ reflection and $0.333 \%$ for the $200_{\mathrm{M}}$ reflection. These anisotropic microstrains are well known to originate from lattice mismatch at domain walls (Choi et al., 2010; Daniels et al., 2006).

Further analysis by high-resolution neutron diffraction is shown in Fig. 1(b), which illustrates the Rietveld refinement with the same structure model used for the high-resolution synchrotron X-ray diffraction data. The threefold splitting of the $00 l$ reflections can be observed and is modelled adequately by the fit [Fig. 1(b), inset III]. The authors have published this structure model, albeit with less detail, for the same material (Mgbemere et al., 2012). Other studies reached the same conclusion for similar KNN compositions, although without clear reflection splitting (Benčan et al., 2009; Mgbemere et al., 2016; Shiratori et al., 2005; Tellier et al., 2009; Baker et al., 2009; Liu et al., 2016). The present work appears to resolve such reflection splitting for the first time, which is attributed to the high angular resolution of the X-ray and neutron diffraction data.

Since $\mathrm{KNN}-\mathrm{Li}_{2} \mathrm{Mn}$ showed the features of $P m$ symmetry clearly and distinctively, its temperature dependence was investigated in further detail using neutron diffraction. A previous X-ray study suggested the existence of phase transformations to tetragonal at $\sim 423 \mathrm{~K}$ and to a paraelectric cubic phase at $\sim 723 \mathrm{~K}$ (Mgbemere et al., 2012). Therefore, data were obtained for the material at temperatures within the 
tetragonal range (523 K) (Fig. $2 a$ ) and above the Curie temperature (773 K) (Fig. $2 b$ ).

Surprisingly, it was not possible to refine the data above the Curie temperature using a cubic space group. All higherindexed reflections show clear splitting. The most appropriate method of describing the structure is with the use of two identical cubic phases of different lattice parameters. Fig. 2(b) shows the Rietveld refinement at $773 \mathrm{~K}$ with two cubic phases, $P_{\mathrm{C}}^{1}\left[a_{1}=3.98997(7) \AA\right]$ and $P_{\mathrm{C}}^{2}\left[a_{2}=3.98337(10) \AA\right]$. The distinct splitting of the highest-indexed reflection, $422_{\mathrm{C}}$ [arrows $P_{\mathrm{C}}^{1}$ and $P_{\mathrm{C}}^{2}$ in the inset in Fig. 2(b)] clearly shows that the material is composed of more than a single phase.

Closer examination of the results for the tetragonal stability region at $523 \mathrm{~K}$ shows that here too the measured data can be described only by the existence of two phases $\left(P_{\mathrm{T}}^{1}\right.$ and $P_{\mathrm{T}}^{2}$; Fig. 2a). In this case, two tetragonal $P 4 \mathrm{~mm}$ phases with different lattice parameters allow adequate modelling of the observed data. In light of these results for the measurements at 523 and $773 \mathrm{~K}$, the measurements at room temperature require reassessment. The literature (Baker et al., 2009;

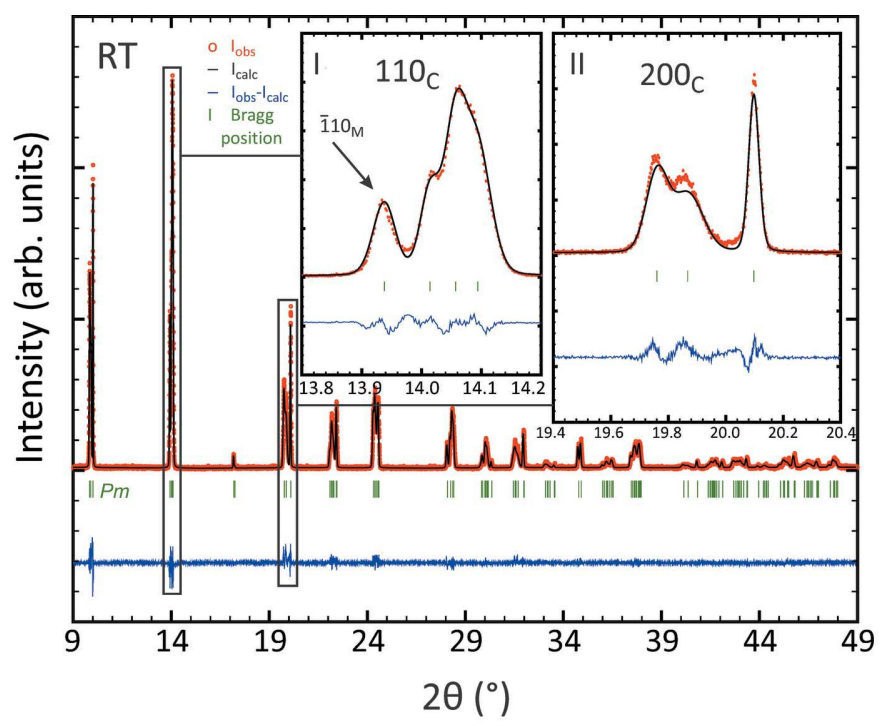

(a)

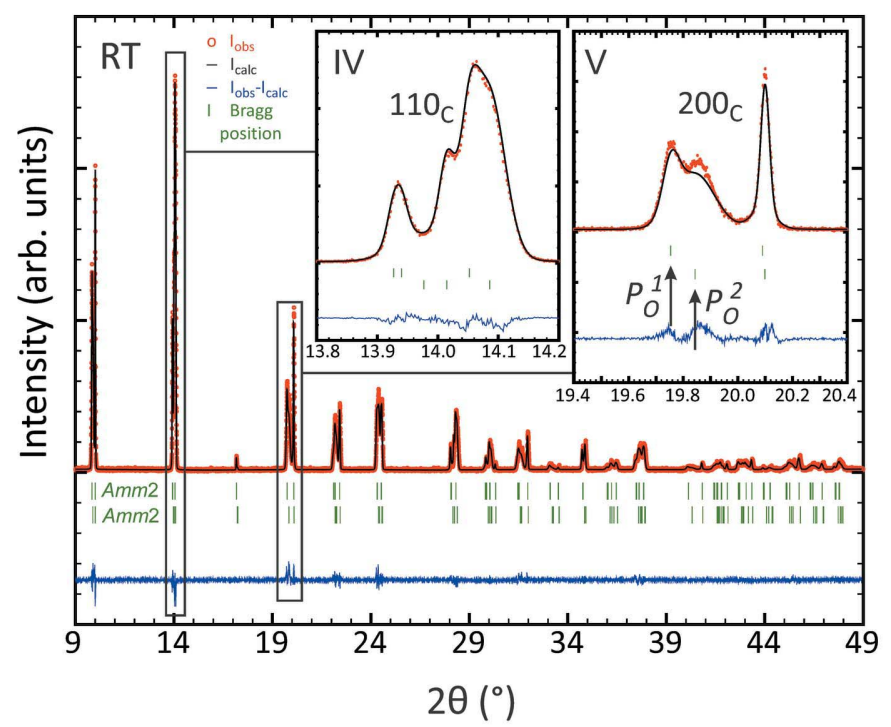

(c)

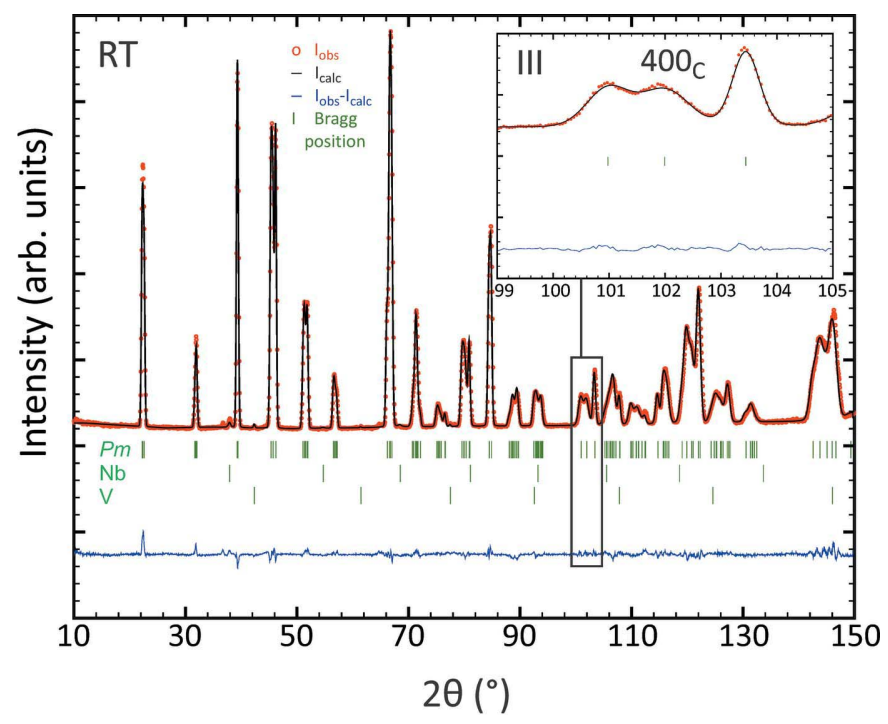

(b)

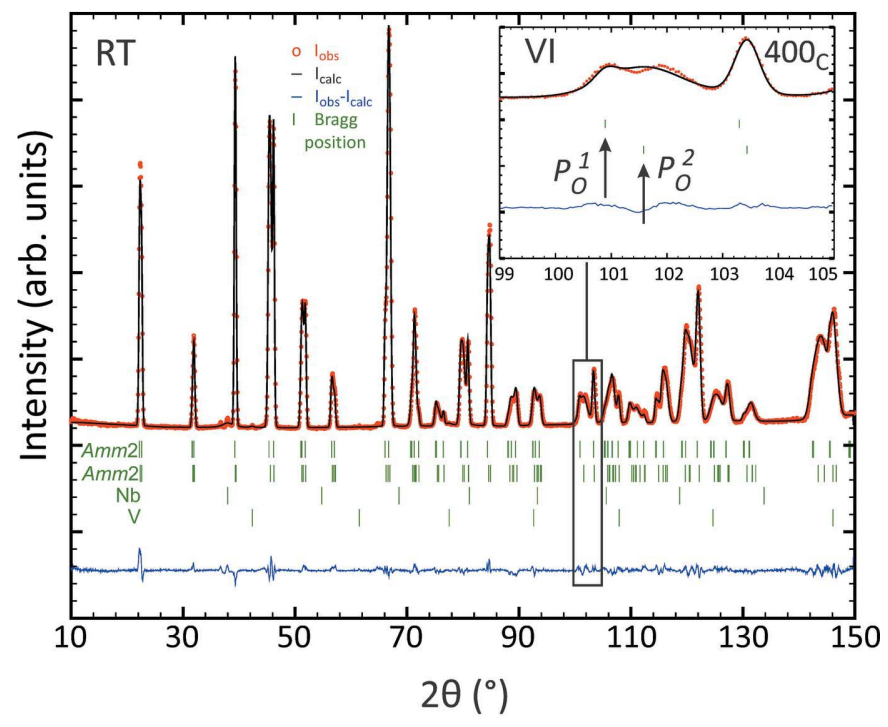

(d)

Figure 1

Rietveld refinement of $(a),(c)$ high-resolution synchrotron X-ray diffraction data and $(b),(d)$ high-resolution neutron diffraction data for $\mathrm{KNN}-\mathrm{Li}_{2} \mathrm{Mn}$. The red dots show the measurements, the black curves indicate the calculated diffraction patterns and the blue curves show the difference of fit. The green vertical lines indicate the positions of the Bragg reflections. $(a)$ and $(b)$ show the fit for a single-phase Pm structure model. Inset I shows the $110_{\mathrm{C}}$ reflection with clear splitting into the $110_{\mathrm{C}}$ and the $111_{\mathrm{M}}$ reflections (arrow) due to the monoclinic angle. The reflection clearly is composed of four reflections instead of three when space group $A m m 2$ is used. Inset II depicts the $200_{\mathrm{C}}$ reflection with clear threefold splitting due to the three different monoclinic primitive axes. Inset III shows clear threefold splitting $\left(101,102\right.$ and $\left.103.5^{\circ} 2 \theta\right)$ of the $400_{\mathrm{C}}$ reflection. $(c)$ and $(d)$ show a fit for a structure model with two phases of space group Amm2. Inset IV shows fourfold splitting of the $110_{\mathrm{C}}$ reflection; inset V shows threefold splitting of the $200_{\mathrm{C}}$ reflection; inset VI shows threefold splitting of the $400_{\mathrm{C}}$ reflection. As the niobium heat shield gives rise to a clearly visible 110 reflection at about $38.5^{\circ}$ and the vanadium sample container gives rise to a 110 reflection at about $42.5^{\circ}$, these phases are included in the fitting. 
Table 2

Details of the Rietveld refinements for the different temperatures and structure models.

The number of refined parameters is given for every discussed structure model, temperature and measurement. The numbers are split into groups of common (co.) and individual (in.) sets of parameters. Sums in brackets give the individual parameters for the two phases $\left(\mathrm{ph}_{1}, \mathrm{ph}_{2}\right)$ of the two-phase structure models separated by the number of constrained parameters for both phases. The fit agreement parameter $\left(\chi^{2}\right)$ and the profile $\left(R_{\mathrm{p}}\right)$ and weighted profile $R$ factors $\left(R_{\mathrm{wp}}\right)$ are given as well.

\begin{tabular}{|c|c|c|c|c|c|c|}
\hline \multirow{2}{*}{$\begin{array}{l}\text { Temperature } \\
\text { Source }\end{array}$} & \multicolumn{4}{|c|}{ Room temperature } & \multirow{2}{*}{$\begin{array}{l}523 \mathrm{~K} \\
\mathrm{ND}\end{array}$} & \multirow{2}{*}{$\begin{array}{l}773 \mathrm{~K} \\
\mathrm{ND}\end{array}$} \\
\hline & XRD & & ND & & & \\
\hline Phases & $P m$ & $A m m 2+A m m 2$ & $P m$ & $A m m 2+A m m 2$ & $P 4 m m+P 4 m m$ & $P m 3 m+P m 3 m$ \\
\hline \multirow[t]{2}{*}{ No. of parameters } & \multirow{2}{*}{$\begin{array}{l}\text { in. co. } \\
31+11\end{array}$} & $\begin{array}{cc}\text { (in.) co. } & \text { co. } \\
(15+2+15)+11\end{array}$ & \multirow{2}{*}{$\begin{array}{l}\text { in. } \quad \text { co. } \\
32+15\end{array}$} & $\begin{array}{c}\text { (in.) co. } \\
(16+1+16)+15\end{array}$ & $\begin{array}{cr}\text { (in.) } & \text { co. } \\
(13+2+13)+13\end{array}$ & $\begin{array}{c}\text { (in.) co. } \\
(6+1+6)+13\end{array}$ \\
\hline & & $\mathrm{ph}_{1} \quad \mathrm{ph}_{2}$ & & $\mathrm{ph}_{1} \quad \mathrm{ph}_{2}$ & $\mathrm{ph}_{1} \quad \mathrm{ph}_{2}$ & $\mathrm{ph}_{1} \quad \mathrm{ph}_{2}$ \\
\hline$R_{\mathrm{wp}}(\%)$ & 15.24 & 16.31 & 4.44 & 4.34 & 5.61 & 6.36 \\
\hline$\chi^{2}$ & 1.74 & 2.00 & 7.89 & 7.53 & 11.99 & 15.37 \\
\hline
\end{tabular}

Mgbemere et al., 2012) and earlier observations suggest that the phase transformation sequence in $\mathrm{KNN}-\mathrm{Li}_{2} \mathrm{Mn}$ should be

$$
A m m 2 \stackrel{423 \mathrm{~K}}{\rightarrow} P 4 m m \stackrel{723 \mathrm{~K}}{\rightarrow} P m \overline{3} m
$$

Careful revision of the refinements reveals that the roomtemperature data can be modelled with two similar phases as well: $P_{\mathrm{O}}^{1}$ and $P_{\mathrm{O}}^{2}$. Accordingly, the monoclinic phase is not necessarily needed to refine the structure.

The use of two Amm 2 phases also results in good agreement between fit and observations for both high-resolution synchrotron and neutron diffraction data $\left(P_{\mathrm{O}}^{1}\right.$ and $P_{\mathrm{O}}^{2} ;$ Figs. $1 c$ and $1 d)$. While the refinement with the synchrotron data (Fig. 1c) appears similar to that using Pm (Fig. 1a), the model is not capable of reproducing the threefold splitting of the
$400_{\mathrm{C}}$ reflection with neutron diffraction (Fig. $1 d$ ), resulting in a slightly inferior fit. Since orthorhombic phases exhibit fewer degrees of freedom than monoclinic phases, the fit of the pronounced anisotropic reflection broadening is limited.

Tables 1-3 and the supporting information give details of the refinements for the different temperatures and structure models. The $R$ and $\chi^{2}$ values for the synchrotron refinements at room temperature reveal that the single-phase model is slightly better, while the results for the neutron refinements suggest that the two-phase model is better. With similar numbers of refined parameters, no clear conclusion can be drawn from the refinement results, on the basis of agreement factors. Since the number of calculated reflections is higher for the two-phase model while maintaining a similar result, the pure assessment of the refinements would favour the single-

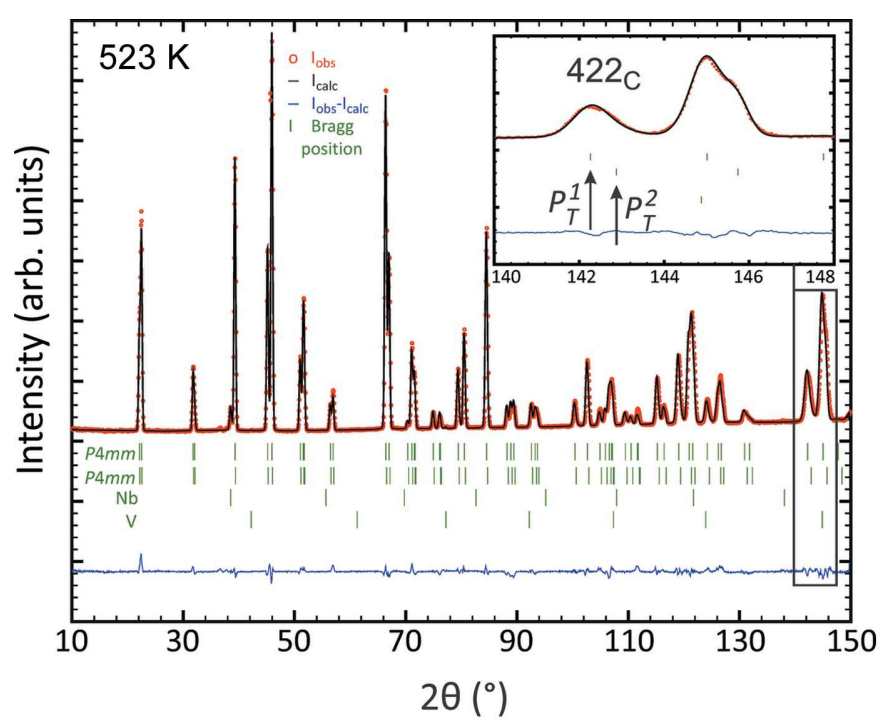

Figure 2

(a)

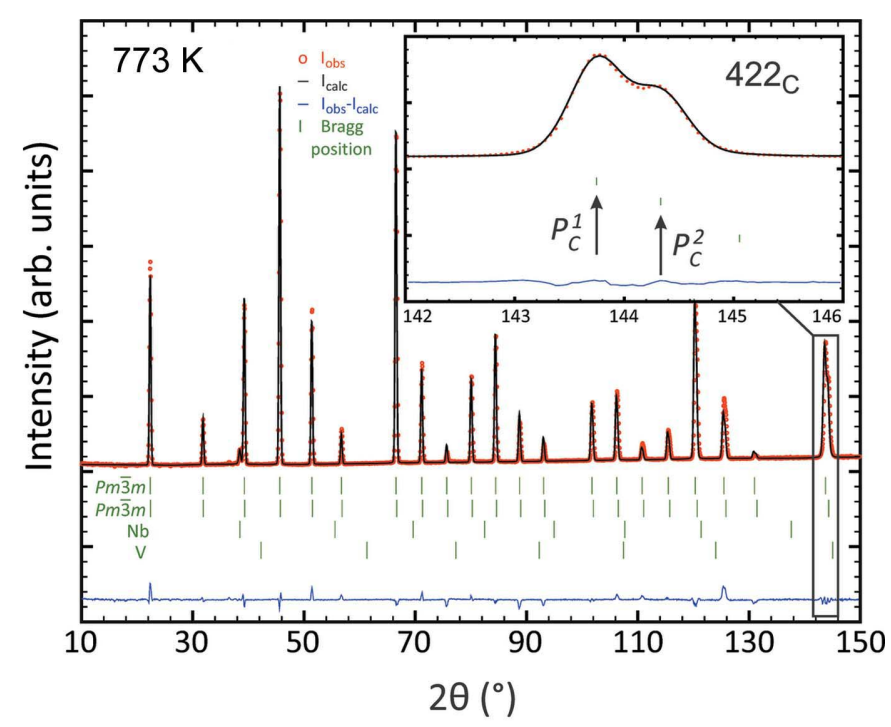

(b)

refinement of high-resolution neutron diffraction data of $\mathrm{KNN}-\mathrm{Li}_{2} \mathrm{Mn}$ at $(a) 523 \mathrm{~K}$ and $(b) 773 \mathrm{~K}$. The red dots show the measurements, the black curves indicate the calculated diffraction patterns and the blue curves show the difference of fit. The green vertical lines indicate the positions of the Bragg reflections. The insets show the clear splitting of the $422_{\mathrm{C}}$ reflection. As the niobium heat shield gives rise to a clearly visible 110 reflection at about $38.5^{\circ}$ and the vanadium sample container gives rise to a 110 reflection at about $42.5^{\circ}$, these phases are included in the fitting. 
Table 3

Lattice parameters, unit-cell angle $\beta$ and unit-cell volume of all phases used in the refinements.

\begin{tabular}{llllllll}
\hline & $P m$ & $A m m 2$ & $A m m 2$ & $P 4 m m$ & $P 4 m m$ & $P m \overline{3} m$ & $P m \overline{3} m$ \\
\hline$a(\AA)$ & $4.01035(5)$ & $3.94590(5)$ & $3.94200(5)$ & $3.96592(8)$ & $3.95807(10)$ & $3.99001(5)$ & $3.98346(7)$ \\
$b(\AA)$ & $3.98782(5)$ & $5.6366(2)$ & $5.66580(15)$ & $3.96592(8)$ & $3.95807(10)$ & $3.99001(5)$ & $3.98346(7)$ \\
$c(\AA)$ & $3.94398(4)$ & $5.64259(20)$ & $5.67416(10)$ & $4.02904(7)$ & $4.0220(2)$ & $3.99001(5)$ & $3.98346(7)$ \\
$\beta\left({ }^{\circ}\right)$ & $89.6915(6)$ & 90 & 90 & 90 & 90 & 90 & 90 \\
$V\left(\AA^{3}\right)$ & $63.0736(13)$ & $125.500(7)$ & $126.730(4)$ & $63.371(2)$ & $63.009(4)$ & $63.5217(13)$ & $63.2093(20)$ \\
\hline
\end{tabular}

Table 4

EPMA-WDS results†.

$x$ is the concentration calculated from the measurement in $\mathrm{mol} \%$.

\begin{tabular}{|c|c|c|c|c|c|c|c|c|c|c|}
\hline \multirow[b]{3}{*}{ Element } & \multicolumn{6}{|l|}{$i, t$} & & & & \\
\hline & \multicolumn{2}{|c|}{$22 \mathrm{nA}, 20 \mathrm{~s}$} & \multicolumn{2}{|c|}{$40 \mathrm{nA}, 40 \mathrm{~ms}$} & \multicolumn{2}{|c|}{$80 \mathrm{nA}, 25 \mathrm{~ms}$} & \multicolumn{2}{|c|}{ Image analyses } & \multicolumn{2}{|c|}{ Rietveld fit } \\
\hline & $\mathrm{Na}$ & $\mathrm{K}$ & $\mathrm{Na}$ & $\mathrm{K}$ & $\mathrm{Na}$ & $\mathrm{K}$ & $\begin{array}{l}P_{\mathrm{O}}^{1} \\
\text { (Na-rich) }\end{array}$ & $\begin{array}{l}P_{\mathrm{O}}^{2} \\
\text { (K-rich) }\end{array}$ & $\begin{array}{l}P_{\mathrm{O}}^{1} \\
\text { (Na-rich) }\end{array}$ & $\begin{array}{l}P_{\mathrm{O}}^{2} \\
\text { (K-rich) }\end{array}$ \\
\hline$x(\mathrm{~mol} \%)$ & 9.78 & 9.62 & 11.61 & 10.15 & 10.83 & 9.31 & & & & \\
\hline FWHM (\%) & - & - & 1.72 & 1.18 & 1.37 & 1.05 & & & & \\
\hline Mean $x(\mathrm{~mol} \%)$ & 9.70 & & 10.46 & & 9.94 & & & & & \\
\hline Mean FWHM (\%) & - & & 1.32 & & 1.46 & & & & & \\
\hline Fraction (\%) & 50.41 & 49.59 & 55.53 & 48.55 & 54.46 & 46.82 & 52.78 & 47.21 & $52.5(5)$ & $47.5(5)$ \\
\hline FWHM (\%) & - & - & 8.20 & 5.65 & 6.89 & 5.29 & & & & \\
\hline
\end{tabular}

$\dagger$ The values measured at $22 \mathrm{nA}$ were obtained from 30 selected points and summed. The values measured at 40 and $80 \mathrm{nA}$ are total sums for scans over the same $60 \times 60 \mu \mathrm{m}$ area with a step width of $0.2 \mu \mathrm{m}$. Image analyses were performed on the Na-rich and K-rich distributions ( $40 \mathrm{nA}, 40 \mathrm{~ms}$ ) using the data in Fig. 3.
Although the coexistence of two orthorhombic Amm2 phases at room temperature seems more likely, it is clear that it is not possible to judge a structure model from fit parameters by implication (Toby, 2006). In fact, careful assessment of the microstructure by complementary techniques, such as electron microscopy, followed by conclusions based on physically reasonable arguments, would be advantageous.

\subsection{Electron microscopy}

In order to determine the origin of this phase coexistence and to correlate the crystallography with the microstructure, the local microstructure was investigated using electron probe microanalysis with wavelength-dispersive spectroscopy (EPMA-WDS). Figs. 3 and 4 illustrate the results of two scans of the same area using different sample currents and dwell times.

When the scan was conducted at a phase model. With increasing temperature, the quality of the neutron refinements decreases together with the number of fit parameters.

Considering only the agreement factors and the appearance of the fits together with the number of calculated reflections, single-phase $P m$ appears to be the correct structure model. Further, given the common symmetry evolutions with temperature, it is highly unlikely that coexisting phases of high symmetry transform to a single low-symmetry phase at lower temperatures. Usually, symmetry increases with increasing temperature owing to the Gibbs free energy dependence. In principle, there are several possible scenarios in which coexisting phases transform to a single phase with decreasing temperature. Superlattices, spinodal decompositions and subsolidus miscibility gaps are such cases. lower sample current of $40 \mathrm{nA}$, a bimodal distribution of the $A$-site cations was clearly evident. When the sample current was increased to $80 \mathrm{nA}$, as shown in Fig. 3, the bimodal distribution of $A$-site cations was even more pronounced, indicating this to be a bulk phenomenon rather than a surface effect or an outcome of thermal etching. While potassium is enriched at the grain boundaries, sodium has a higher concentration in the bulk of the grains. The distribution map also exhibits regions where neither of the two elements can be found. These correspond to pores in the sample, which appear as either black holes in the scanning electron microscope image or dark-grey areas between the grains.

For further clarity, a second scan at a higher sample current and shorter dwell time was obtained in order to map oxygen and manganese (Fig. 4). The results show that the sample
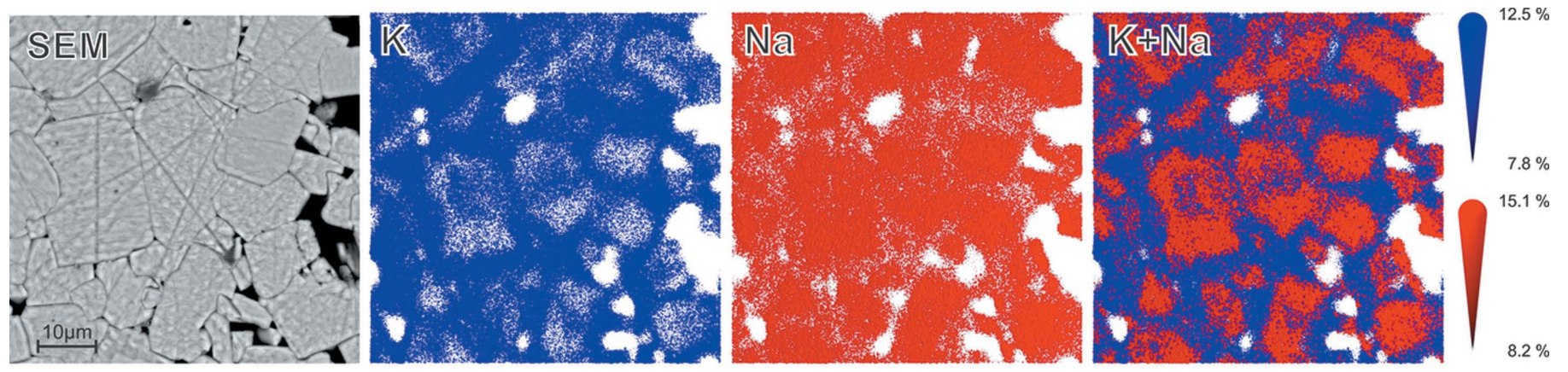

Figure 3

EPMA-WDS scans of a $60 \times 60 \mu \mathrm{m}$ area with elemental mapping of $\mathrm{K}$ and $\mathrm{Na}$ as well as the corresponding scanning electron microscopy image. At an accelerating voltage of $20 \mathrm{kV}$, the sample current was adjusted to $80 \mathrm{nA}$, with a dwell time of $25 \mathrm{~ms}$ per point and a step width of $0.2 \mu \mathrm{m}$ for the mapping. Individual scan points are represented by spheres with diameters proportional to the mol\% of the elements. 
exhibits a continuous oxygen matrix and discrete particles of manganese oxide. It is clear that the manganese content was higher than the manganese solubility. Consequently, the manganese dopant is distributed uniformly throughout the matrix grains and shows no correlation with the $A$-site distribution.

The same areas depicted in Figs. 3 and 4 were scanned (30 points) at a sample current of $22 \mathrm{nA}$ and a dwell time of $20 \mathrm{~s}$ in order to compare spatial distributions and counting statistics. Table 4 summarizes the analysis of the two mapping scans at the selected points and compares them with the results from the Rietveld refinement and image analyses for the Na-rich and K-rich distributions (40 nA, $40 \mathrm{~ms}$ ) from Fig. 3.

The EPMA-WDS results clearly show that $\mathrm{Na}$ and $\mathrm{K}$ are segregated into different regions within the microstructure. $\mathrm{Na}$ is mostly present within the grains and $\mathrm{K}$ is predominantly located at the grain boundaries. The image analyses and the results from Rietveld refinement for the two orthorhombic phases $P_{\mathrm{O}}^{1}$ and $P_{\mathrm{O}}^{2}$ show excellent agreement, strengthening the concept of coexistence of two similar phases with different $\mathrm{Na} / \mathrm{K}$ ratios over the entire temperature range.

\section{Discussion}

In summary, the EPMA-WDS, X-ray diffraction and neutron diffraction data show segregation at the grain boundaries, resulting in two different chemical compositions $(\mathrm{Na} / \mathrm{K})$ at the grain boundaries and in the core of the grains. A similar coreshell microstructure has been reported before for KNN (Zhen \& Li, 2007, 2006), doped barium titanate (Jeon et al., 2012; Jeon \& Kang, 2013) and barium strontium titanate (Kim \&
Kang, 1999). Many other important perovskite materials may potentially show similar behaviour as they either are doped or have $A$ - or $B$-site substitution [e.g. PZT (Hammer \& Hoffmann, 1998), BT (Jeon et al., 2012; Jeon \& Kang, 2013), strontium titanate (ST) (Chung et al., 2005), $\mathrm{Ba}\left(\mathrm{Ti}_{0.8} \mathrm{Zr}_{0.2}\right) \mathrm{O}_{3}-$ $x\left(\mathrm{Ba}_{0.7} \mathrm{Ca}_{0.3}\right) \mathrm{TiO}_{3}(\mathrm{BCT}-\mathrm{BZT})$ (Wang et al., 2013), BNT (Liu \& Tan, 2016), BNT-BT (Hinterstein et al., 2015), BNT-BTKNN (Schmitt \& Kleebe, 2010), BNT-ST (Koruza et al., 2016; Acosta et al., 2015; Kim \& Kang, 1999), KNN (Zhen \& Li, 2007; Wang et al., 2007) and $\mathrm{Ba}_{0.5} \mathrm{Sr}_{0.5} \mathrm{Co}_{0.8} \mathrm{Fe}_{0.2} \mathrm{O}_{3-\delta}$ (Zhou et al., 2011)]. Accordingly, this behaviour and its origin are of considerable interest.

Segregation of ions to grain surfaces occurs as a result of lattice stress (Idris et al., 2012). This can take place during both calcination and sintering, although the latter is more likely owing to the greater bulk density of the ceramic and hence greater volumetric stress concentration. As the ionic radii of $\mathrm{Na}$ and $\mathrm{K}$ are significantly different (in sixfold coordination, $K$ is $>30 \%$ larger than $\mathrm{Na}$; Shannon, 1976), $\mathrm{K}$ would be expected to exhibit faster and a greater amount of segregation, resulting in enrichment of $\mathrm{Na}$ in the grain centre and enrichment of $\mathrm{K}$ at the grain boundaries. This phenomenon has been reported for $\mathrm{BaTiO}_{3}-\mathrm{SrTiO}_{3}$ (Kim \& Kang, 1999) and BT (Jeon et al., 2012). Although Malič et al. (2008) reported that Na diffusion was more rapid than that of $\mathrm{K}$ for the compounds $\mathrm{K}_{2} \mathrm{CO}_{3}$, $\mathrm{Na}_{2} \mathrm{CO}_{3}$ and mixtures of the two in apposition with $\mathrm{Nb}_{2} \mathrm{O}_{5}$, these data reflect chemical gradient diffusion rather than segregation. However, they do highlight the potential effects of kinetics in these processes. In this case, while segregation would enrich the grain boundaries with $\mathrm{K}$, diffusion under a chemical gradient would enrich them with Na. The core-shell
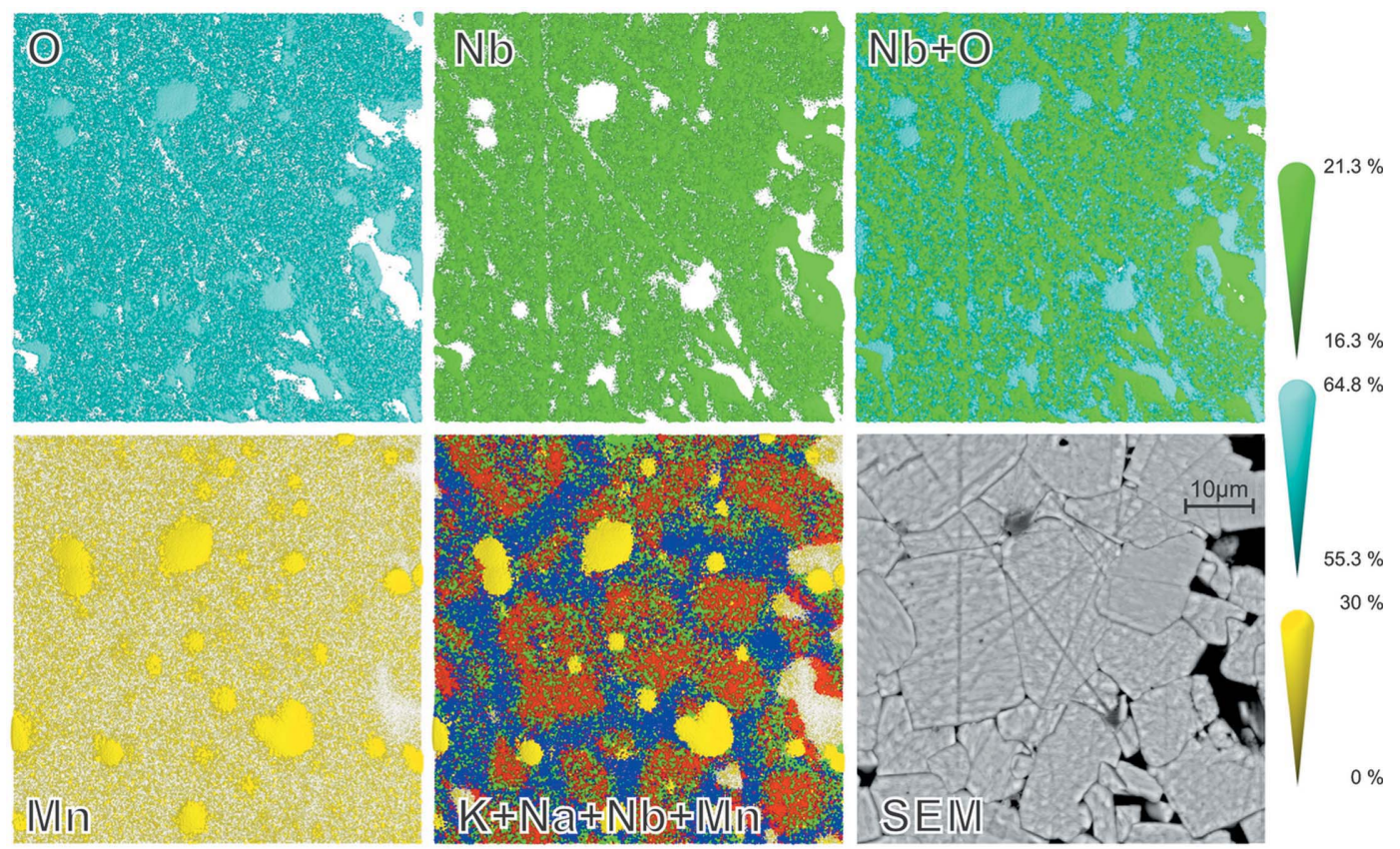

Figure 4

EPMA-WDS scans of a $60 \times 60 \mu \mathrm{m}$ area with elemental mapping of $\mathrm{O}, \mathrm{Nb}$ and $\mathrm{Mn}$ together with $\mathrm{K}$ and $\mathrm{Na}$ as well as the corresponding scanning electron microscopy image. At an accelerating voltage of $20 \mathrm{kV}$, the sample current was adjusted to $80 \mathrm{nA}$, with a dwell time of $25 \mathrm{~ms}$ per point and a step width of $0.2 \mu \mathrm{m}$ for the mapping. Individual scan points are represented by spheres with diameters proportional to the mol\% of the elements. 
distribution in the present work is limited spatially to the grain size, so it is likely that the driving force for this distribution is differential segregation of the two alkalis that occurred during the latter stage of densification and resulted in greater segregation of the larger alkali ion.

In contrast, segregation from kinetic effects during sintering is quite probable owing to volatilization. The volatilization rate of $\mathrm{Na}$ can be concluded to be greater than that of $\mathrm{K}$ owing to the observation of the formation of K-rich surface layers (Acker et al., 2014; Zhen \& Li, 2006, 2007). The microstructure shown in Figs. 3 and 4 supports the view that segregation occurred during the sintering process. It also is likely that the extensive grain growth was assisted by the concentration of the alkalis both in the bulk and at the surfaces since these ions are well known as high-diffusivity fluxes for oxides.

Thermodynamically, segregation may arise from the existence of charged boundaries and the formation of adjacent space charge (Chiang \& Takagi, 1990; Sutton \& Balluffi, 1996; Chiang et al., 1997). However, the typical width of concentration gradients due to space charge is in the range of tens of nanometres, while that observed in Fig. 3 is of the order of several micrometres. Another source of thermodynamic segregation has been designated as a 'complexion', where a thin segregated (adsorption) film at the interfaces may form because of interfacial energy minimization (Cantwell et al., 2014; Dillon et al., 2007; Rheinheimer \& Hoffmann, 2016). However, since these films usually are atomically disordered and have a width of only a few nanometres, this phenomenon is unlikely.

Finally, a plausible kinetic and thermodynamic basis for the observed segregation could be the miscibility gap that is observed in some systems containing $\mathrm{Na}$ and $\mathrm{K}$, including $\mathrm{NaCl}-\mathrm{KCl}$ (Thompson \& Waldbaum, 1969; Burton \& van de Walle, 2006), $\mathrm{NaAlSi}_{3} \mathrm{O}_{8}-\mathrm{KAlSi}_{3} \mathrm{O}_{8}$ (Yund \& Tullis, 1983), $\mathrm{NaNO}_{3}-\mathrm{KNO}_{3}$ (Levin et al., 1964) and $\mathrm{Na}_{3} \mathrm{~K}\left(\mathrm{AlSiO}_{4}\right)_{4}-$ $\mathrm{K}_{4}\left(\mathrm{AlSiO}_{4}\right)_{4}($ Ferry \& Blencoe, 1978), although it has not been reported for the $\mathrm{NaNbO}_{3}-\mathrm{KNbO}_{3}$ system (Shirane et al., 1954; Ahtee \& Hewat, 1978). Such a subsolidus miscibility gap could result in the thermodynamic stability of two different material compositions. That is, a homogenous single-phase solid solution at the sintering temperature would undergo transformation into two solid solution end members of different compositions upon cooling. These microstructures are indicative of the incomplete phase separation of the Na-rich and K-rich conjugate phases, which would explain both the appearance and the evolution of the observed microstructures.

\section{Conclusions}

The present work shows that local variations in the substituted species distribution can lead to phenomena that can be explained by monoclinic average symmetry as well as phase coexistence of higher-symmetry phases. Only a temperaturedependent X-ray and neutron diffraction study in conjunction with precise wavelength dispersive spectroscopy elemental analyses are able to demonstrate local differences in concen- trations of the substituted species, resulting in a bimodal distribution of concentrations with different crystal structures.

This represents an extreme case of segregation (in the generic sense of this term) on the micrometre scale. However, intermediate chemical segregation down to the unit-cell level is conceivable, although this would be uncertain at such a scale. Many perovskites and other materials are known to show such segregation at the interfaces, which may be caused by, inter alia, charged boundary cores or interfacial stresses. The typical scale of interfacial segregation in these materials at high temperatures is of the order of 10-500 nm (Chiang \& Takagi, 1990; Bäurer et al., 2010; Rheinheimer \& Hoffmann, 2016).

The present work confirms that the use of Rietveld refinement alone can make structure determinations challenging. However, the underlying mechanisms of the extraordinary properties of some functional materials originate not just in their structures but also in their complex microstructures. Consequently, knowledge of both features of materials may be essential for the exploitation and development of their functionalities.

\section{Funding information}

M. Hinterstein acknowledges funding from the Deutsche Forschungsgemeinschaft (DFG) for the Emmy Noether Research Group HI1867/1-1. M. Hoffman acknowledges Australian Research Council Discovery Project DP150104649.

\section{References}

Acker, J., Kungl, H., Schierholz, R., Wagner, S., Eichel, R.-A. \& Hoffmann, M. J. (2014). J. Eur. Ceram. Soc. 34, 4213-4221.

Acosta, M., Schmitt, L. A., Molina-Luna, L., Scherrer, M. C., Brilz, M., Webber, K. G., Deluca, M., Kleebe, H.-J., Rödel, J. \& Donner, W. (2015). J. Am. Ceram. Soc. 98, 3405-3422.

Ahn, Z. S. \& Schulze, W. A. (1987). J. Am. Ceram. Soc. 70, C-18-C-21. Ahtee, M. \& Hewat, A. W. (1978). Acta Cryst. A34, 309-317.

Aksel, E., Forrester, J. S., Jones, J. L., Thomas, P. A., Page, K. \& Suchomel, M. R. (2011). Appl. Phys. Lett. 98, 152901.

Baker, D. W., Thomas, P. A., Zhang, N. \& Glazer, A. M. (2009). Appl. Phys. Lett. 95, 091903.

Bäurer, M., Shih, S.-J., Bishop, C., Harmer, M. P., Cockayne, D. \& Hoffmann, M. J. (2010). Acta Mater. 58, 290-300.

Benčan, A., Tchernychova, E., Godec, M., Fisher, J. \& Kosec, M. (2009). Microsc. Microanal. 15, 435-440.

Bomlai, P., Wichianrat, P., Muensit, S. \& Milne, S. J. (2007). J. Am. Ceram. Soc. 90, 1650-1655.

Boysen, H. (2005). Z. Kristallogr. 220, 726-734.

Boysen, H. (2007). J. Phys. Condens. Matter, 19, 275206.

Burton, B. P. \& van de Walle, A. (2006). Chem. Geol. 225, 222-229.

Cantwell, P. R., Tang, M., Dillon, S. J., Luo, J., Rohrer, G. S. \& Harmer, M. P. (2014). Acta Mater. 62, 1-48.

Chiang, Y., Birnie, D. P. \& Kingery, W. D. (1997). Physical Ceramics. New York: J. Wiley.

Chiang, Y.-M. \& Takagi, T. (1990). J. Am. Ceram. Soc. 73, 3278-3285.

Choi, Y.-K., Hoshina, T., Takeda, H., Teranishi, T. \& Tsurumi, T. (2010). Appl. Phys. Lett. 97, 212907.

Chung, S.-Y., Lee, B.-K. \& Kang, S. L. (2005). J. Am. Ceram. Soc. 81, 3016-3018.

Cox, D. E., Noheda, B., Shirane, G., Uesu, Y., Fujishiro, K. \& Yamada, Y. (2001). Appl. Phys. Lett. 79, 400-402. 
Daniels, J. E., Jones, J. L. \& Finlayson, T. R. (2006). J. Phys. D Appl. Phys. 39, 5294-5299.

Dillon, S. J., Tang, M., Carter, W. C. \& Harmer, M. P. (2007). Acta Mater. 55, 6208-6218.

Ferry, J. M. \& Blencoe, J. G. (1978). Am. Mineral. 63, 1225-1240.

Forsbergh, P. W. (1949). Phys. Rev. 76, 1187-1201.

Gorfman, S. \& Thomas, P. A. (2010). J. Appl. Cryst. 43, 1409-1414.

Grinberg, I., Cooper, V. R. \& Rappe, A. M. (2002). Nature, 419, 909911.

Hammer, M. \& Hoffmann, M. J. (1998). J. Am. Ceram. Soc. 81, $3277-$ 3284.

Hinterstein, M., Schmitt, L. A., Hoelzel, M., Jo, W., Rödel, J., Kleebe, H.-J. \& Hoffman, M. (2015). Appl. Phys. Lett. 106, 222904.

Hoelzel, M., Senyshyn, A., Juenke, N., Boysen, H., Schmahl, W. \& Fuess, H. (2012). Nucl. Instrum. Methods Phys. Res. A, 667, 32-37.

Idris, M. A., Bak, T., Li, S. \& Nowotny, J. (2012). J. Phys. Chem. C, 116, 10950-10958.

Jaffe, B., Cook, W. R. \& Jaffe, H. (1971). Piezoelectric Ceramics. London: Academic Press.

Jeon, S.-C. \& Kang, S. L. (2013). Appl. Phys. Lett. 102, 112915.

Jeon, S.-C., Lee, C.-S. \& Kang, S. L. (2012). J. Am. Ceram. Soc. 95, 2435-2438.

Kay, H. F. \& Vousden, P. (1949). London Edinb. Dubl. Philos. Mag. J. Sci. 40, 1019-1040.

Keeble, D. S., Benabdallah, F., Thomas, P. A., Maglione, M. \& Kreisel, J. (2013). Appl. Phys. Lett. 102, 092903.

Kim, J.-S. \& Kang, S. L. (1999). J. Am. Ceram. Soc. 82, 1085-1088.

Knapp, M., Baehtz, C., Ehrenberg, H. \& Fuess, H. (2004). J. Synchrotron Rad. 11, 328-334.

Koruza, J., Rojas, V., Molina-Luna, L., Kunz, U., Duerrschnabel, M., Kleebe, H.-J. \& Acosta, M. (2016). J. Eur. Ceram. Soc. 36, 10091016.

La-Orauttapong, D., Noheda, B., Ye, Z.-G., Gehring, P. M., Toulouse, J., Cox, D. E. \& Shirane, G. (2002). Phys. Rev. B, 65, 144101.

Levin, E. M., Robbins, C. R. \& McMurdie, H. F. (1964). Phase Diagrams for Ceramists. Columbus: American Ceramic Society.

Liu, L., Knapp, M., Ehrenberg, H., Fang, L., Schmitt, L. A., Fuess, H., Hoelzel, M. \& Hinterstein, M. (2016). J. Appl. Cryst. 49, 574-584.

Liu, X. \& Tan, X. (2016). Adv. Mater. 28, 574-578.

Malič, B., Jenko, D., Holc, J., Hrovat, M. \& Kosec, M. (2008). J. Am. Ceram. Soc. 91, 1916-1922.

Malič, B., Koruza, J., Hreščak, J., Bernard, J., Wang, K., Fisher, J. \& Benčan, A. (2015). Materials (Basel), 8, 8117-8146.

Mgbemere, H. E., Hinterstein, M. \& Schneider, G. A. (2011). J. Appl. Cryst. 44, 1080-1089.

Mgbemere, H. E., Hinterstein, M. \& Schneider, G. A. (2012). J. Eur. Ceram. Soc. 32, 4341-4352.

Mgbemere, H., Schneider, G., Hoelzel, M. \& Hinterstein, M. (2016). J. Appl. Cryst. 49, 891-901.

Mishra, S. K., Pandey, D., Lemmens, H. \& Tendeloo, G. (2001). Phys. Rev. B, 64, 54101 .
Noheda, B., Cox, D. E., Shirane, G., Gonzalo, J. A., Cross, L. E. \& Park, S.-E. (1999). Appl. Phys. Lett. 74, 2059-2061.

Pandey, D. \& Agini, R. (2003). Z. Kristallogr. 218, 1-7.

Ranjan, R., Mishra, S. K. \& Pandey, D. (2002). J. Appl. Phys. 92, 3266.

Ranjan, R., Mishra, S. K., Pandey, D. \& Kennedy, B. (2002). Phys. Rev. $B, \mathbf{6 5}, 1-4$.

Rheinheimer, W. \& Hoffmann, M. J. (2016). Curr. Opin. Solid State Mater. Sci. 20, 286-298.

Rodríguez-Carvajal, J. (1993). Physica B, 192, 55-69.

Schmitt, L. A. \& Kleebe, H.-J. (2010). Funct. Mater. Lett. 03, 5558.

Schneider, C. A., Rasband, W. S. \& Eliceiri, K. W. (2012). Nat. Methods, 9, 671-675.

Schönau, K. A., Schmitt, L. A., Knapp, M., Fuess, H., Eichel, R.-A., Kungl, H. \& Hoffmann, M. J. (2007). Phys. Rev. B, 75, 184117.

Shannon, R. D. (1976). Acta Cryst. A32, 751-767.

Shirane, G., Newnham, R. E. \& Pepinsky, R. (1954). Phys. Rev. 96, 581-588.

Shiratori, Y., Magrez, A. \& Pithan, C. (2005). J. Eur. Ceram. Soc. 25, 2075-2079.

Stephens, P. W. (1999). J. Appl. Cryst. 32, 281-289.

Sutton, A. P. \& Balluffi, R. W. (1996). Interfaces in Crystalline Materials. Oxford: Clarendon Press.

Tellier, J., Malic, B., Dkhil, B., Jenko, D., Cilensek, J. \& Kosec, M. (2009). Solid State Sci. 11, 320-324.

Theissmann, R., Schmitt, L. A., Kling, J., Schierholz, R., Schönau, K. A., Fuess, H., Knapp, M., Kungl, H. \& Hoffmann, M. J. (2007). J. Appl. Phys. 102, 024111.

Thompson, J. B. \& Waldbaum, D. R. (1969). Geochim. Cosmochim. Acta, 33, 671-690.

Thompson, P., Cox, D. E. \& Hastings, J. B. (1987). J. Appl. Cryst. 20, 79-83.

Toby, B. H. (2006). Powder Diffr. 21, 67-70.

Wang, Y. (2007). Phys. Rev. B, 76, 024108.

Wang, Y., Damjanovic, D., Klein, N., Hollenstein, E. \& Setter, N. (2007). J. Am. Ceram. Soc. 90, 3485-3489.

Wang, Z., Zhao, K., Guo, X., Sun, W., Jiang, H., Han, X., Tao, X., Cheng, Z., Zhao, H., Kimura, H., Yuan, G., Yin, J. \& Liu, Z. (2013). J. Mater. Chem. C, 1, 522-530.

Yund, R. A. \& Tullis, J. (1983). Feldspar Mineralogy, Reviews in Mineralogy, Vol. 2. Chelsea: Mineralogical Society of America.

Zhang, N., Yokota, H., Glazer, A. M., Ren, Z., Keen, D. A., Keeble, D. S., Thomas, P. A. \& Ye, Z.-G. (2014). Nat. Commun. 5, 5231.

Zhang, N., Yokota, H., Glazer, A. M. \& Thomas, P. A. (2011a). Acta Cryst. B67, 386-398.

Zhang, N., Yokota, H., Glazer, A. M. \& Thomas, P. A. (2011b). Acta Cryst. B67, 461-466.

Zhen, Y. \& Li, J.-F. (2006). J. Am. Ceram. Soc. 89, 3669-3675.

Zhen, Y. \& Li, J.-F. (2007). J. Am. Ceram. Soc. 90, 3496-3502.

Zhou, W., Liang, F., Shao, Z., Chen, J. \& Zhu, Z. (2011). Sci. Rep. 1, 155 . 